\title{
LEVEL-CROSSING ORDERING OF SEMI-MARKOV PROCESSES AND MARKOV CHAINS
}

\author{
FÁTIMA FERREIRA, ${ }^{*}$ CEMAT and Universidade de Trás os Montes e Alto Douro \\ ANTÓNIO PACHECO, ${ }^{* *}$ CEMAT, CLC, and Instituto Superior Técnico, UTL
}

\begin{abstract}
We extend the definition of level-crossing ordering of stochastic processes, proposed by Irle and Gani (2001), to the case in which the times to exceed levels are compared using an arbitrary stochastic order, and work, in particular, with integral stochastic orders closed for convolution. Using a sample-path approach, we establish level-crossing ordering results for the case in which the slower of the processes involved in the comparison is skip-free to the right. These results are specially useful in simulating processes that are ordered in level crossing, and extend results of Irle and Gani (2001), Irle (2003), and Ferreira and Pacheco (2005) for skip-free-to-the-right discrete-time Markov chains, semi-Markov processes, and continuous-time Markov chains, respectively.
\end{abstract}

Keywords: Integral stochastic order; level-crossing ordering; Markov chain; sample path; semi-Markov process; simulation; stochastic ordering

2000 Mathematics Subject Classification: Primary 60E15; 60K15; 68U20

Secondary 60J10; 60J27

\section{Introduction}

In this paper, we consider the level-crossing ordering of stochastic processes [2], [3], [5]. A process $X$ is said to be slower in level crossing than $Y$ if it takes $X$ stochastically longer to exceed any given level than it does $Y$. The definition for the case in which random variables are compared through the stochastic order usual in distribution was proposed in [5], and will hereby be denoted as level-crossing ordering in the usual sense (or st-sense).

Following this definition, we propose the definition of level-crossing ordering in the $\star$-order sense, where the associated order ' $\leq_{\star}$ ' is an arbitrary stochastic order for random variables, as being the former definition with the times to exceed levels compared in the $\star$-order sense instead of the st-sense. In particular, we will consider orders for nonnegative random variables that, using a terminology due to Whitt [9], belong to the class of integral stochastic orders. An order ' $\leq_{\star}$ ' is an integral stochastic order if, given nonnegative random variables $W$ and $Z$,

$$
W \leq_{\star} Z \Leftrightarrow \mathrm{E}[g(W)] \leq \mathrm{E}[g(Z)] \text { for all } g \in G_{\star}
$$

whenever the expected values exist, where $G_{\star}$ is some set of real functions.

More precisely, we will work with integral stochastic orders ' $\leq_{\star}$ ' such that $G_{\star}$ contains only functions that are increasing (more accurately, nondecreasing) on $\mathbb{R}_{+}=[0, \infty$ ) and, moreover,

Received 24 February 2005; revision received 4 June 2005.

* Postal address: UTAD, Departamento de Matemática, Quinta dos Prados, Apartado 1013, 5001-911 Vila Real, Portugal. Email address: mmferrei@utad.pt

** Postal address: Instituto Superior Técnico, Departamento de Matemática, Av. Rovisco Pais, 1049-001 Lisboa, Portugal. Email address: apacheco@math.ist.utl.pt 
are closed for convolution, i.e. integral stochastic orders for positive random variables with increasing functions closed for convolution, or IPICC orders.

For the sake of completeness, we note that the class of IPICC orders includes, in particular, the usual order ('st') as well as the Laplace transform ('LT'), exponential ('exp'), increasing convex ('icx'), increasing concave ('icv'), moments ('M'), expected value ('EV'), and momentgenerating function ('MG') orders (see, for example, [6] and [8] for details of the definitions and properties of these stochastic orders). In these cases, we can make the following identification of $G_{\star}: G_{\text {st }}$ is the set of all increasing functions, $G_{\mathrm{LT}}=\left\{g_{s}, s>0: g_{s}(x)=-\mathrm{e}^{-s x}\right\}$, $G_{\text {exp }}=\left\{g_{s}, s>0: g_{s}(x)=\mathrm{e}^{s x}\right\}, G_{\text {icx }}$ is the set of increasing convex functions, $G_{\text {icv }}$ is the set of increasing concave functions, $G_{\mathrm{M}}$ is the set of integer-power functions, $G_{\mathrm{EV}}$ is a singular set containing the identity function, and $G_{\mathrm{MG}}=\left\{g_{s}, 0<s<1: g_{s}(x)=s^{x}\right\}$.

In this paper, we derive sets of sufficient conditions for the level-crossing ordering, in the IPICC order senses, of discrete-time Markov chains (DTMCs), continuous-time Markov chains (CTMCs), and semi-Markov processes (SMPs) for the case in which the slower of the two processes involved in the comparison is skip-free to the right. We recall that a path of a stochastic process with ordered state space $I$, order-isomorphic to some bounded or unbounded interval of $\mathbb{Z}$, is said to be skip-free to the right if it does not have jumps up more than one level. Moreover, the stochastic process itself is skip-free to the right if its trajectories are almost surely (a.s.) skip-free to the right.

Our results include results of [5], [3], and the authors' paper [2] for the level-crossing ordering of DTMCs, CTMCs, and SMPs, under the relaxation of some of their assumptions. Our proofs use a sample-path approach (see, for example, [1]) and, thus, are of particular interest for the simulation of stochastic processes ordered in level crossing.

We end the introduction with a brief outline of the paper. In Section 2, we provide an extension of the definition of level-crossing ordering of stochastic processes in the usual sense [5] to other stochastic ordering senses, and introduce some notation and basic properties of the IPICC orders and level-crossing ordering. In Section 3, we establish results for the levelcrossing ordering of two DTMCs in the usual sense. These are used in Section 4 to establish results for the level-crossing ordering of SMPs in the IPICC order senses. Finally, in Section 5, we derive sufficient conditions for the level-crossing ordering of CTMCs in the usual sense.

\section{Preliminaries}

In this section, we introduce a few definitions and some notation. In addition, we provide useful results on the level-crossing ordering of stochastic processes and on integral stochastic orders.

We let $\Gamma$ denote the set of natural numbers $\mathbb{N}=\{0,1,2, \ldots\}$, positive integers $\mathbb{N}_{+}=$ $\{1,2, \ldots\}$, or real nonnegative numbers $\mathbb{R}_{+}=[0, \infty)$. Given a state space $I$ of real numbers, $A \subseteq \mathbb{R}$, and $y \in I$, we let $\bar{I}=I \backslash\{\sup I\}$, let $I^{A}=I \cap A$ denote the restriction of $I$ to states in $\bar{A}$, and let $I^{\leq y}:=I^{(-\infty, y]}$ and $I^{\geq y}:==I^{[y, \infty)}$ respectively denote the restrictions of $I$ to states smaller than or equal to $y$ and greater than or equal to $y$. Moreover, if $W=\left(W_{t}\right)_{t \in \Gamma}$ is a stochastic process with state space $I$, we let $R_{y}^{W}$ and $S_{y}^{W}$ respectively denote the hitting times of the sets of values smaller than or equal to $y$ and greater than or equal to $y$, i.e.

$$
R_{y}^{W}=\inf \left\{t \in \Gamma: W_{t} \leq y\right\} \quad \text { and } \quad S_{y}^{W}=\inf \left\{t \in \Gamma: W_{t} \geq y\right\},
$$

where inf $\varnothing \equiv \infty$. Finally, to introduce the definition of level-crossing ordering of stochastic processes, we let $S_{x, y}^{W}$ denote the hitting time of the set of values greater than or equal to $y$ when departing from state $x$. 
Definition 2.1. Let $X=\left(X_{t}\right)_{t \in \Gamma}$ and $Y=\left(Y_{t}\right)_{t \in \Gamma}$ be stochastic processes with common ordered state space $I$, and let ' $\leq_{\star}$ ' be a stochastic order relation for random variables. Then $X$ is said to be slower in level crossing than $Y$ in the $\star$-sense, denoted $X \leq_{\star \mathrm{lc}} Y$, if, for any common initial state $x, S_{x, y}^{Y} \leq_{\star} S_{x, y}^{X}$ for all $y \in I$, i.e.

$$
X \leq_{\star} Y \Leftrightarrow S_{x, y}^{Y} \leq_{\star} S_{x, y}^{X} \quad \text { for all } x, y \in I .
$$

The level-crossing order in the usual sense, ' $\leq$ stlc', is the level-crossing order introduced in [5]. With similar notation, if $W=\left(W_{t}\right)_{t \in \Gamma}$ is a stochastic process with ordered state space $I$, we let $W^{\leq y}$ and $W^{\geq y}, y \in I$, respectively denote the restrictions of the process $W$ to the state spaces $I^{\leq y}$ and $I^{\geq y}$ in such a way that state $y$ is made absorbing and all states of $W$ greater than or equal to $y$, or, respectively, smaller than or equal to $y$, are collapsed into state $y$. That is,

$$
W_{t}^{\leq y}=\left\{\begin{array}{ll}
W_{t}, & t<S_{y}^{W}, \\
y, & t \geq S_{y}^{W},
\end{array} \quad \text { and } \quad W_{t}^{\geq y}= \begin{cases}W_{t}, & t<R_{y}^{W}, \\
y, & t \geq R_{y}^{W} .\end{cases}\right.
$$

With this notation, we have the following two results on the level-crossing ordering of stochastic processes.

Lemma 2.1. If $X=\left(X_{t}\right)_{t \in \Gamma}$ and $Y=\left(Y_{t}\right)_{t \in \Gamma}$ are stochastic processes with ordered state space $I$ and ' $\leq_{\star}$ ' is a stochastic order relation for random variables, then

$$
X \leq_{\star l \mathrm{c}} Y \Leftrightarrow X^{\leq y} \leq_{\star \mathrm{lc}} Y^{\leq y} \quad \text { for all } y \in I .
$$

Proof. Note that if $W=\left(W_{t}\right)_{t \in \Gamma}$ is a stochastic process with ordered state space $I$, then, for each $x, y \in I, S_{x, y}^{W}=S_{x, y}^{W \leq z}$ for all $z \geq \max (x, y)$. As a consequence, if $X=\left(X_{t}\right)_{t \in \Gamma}$ and $Y=\left(Y_{t}\right)_{t \in \Gamma}$ are stochastic processes with state space $I$ and ' $\leq_{\star}$ ' is a stochastic order relation for random variables, then

$$
\begin{aligned}
X \leq_{\star} Y \mathrm{c} Y & \Leftrightarrow S_{x, y}^{Y} \leq_{\star} S_{x, y}^{X} & & \text { for all } x, y \in I \\
& \Leftrightarrow S_{x, y}^{Y \leq z} \leq_{\star} S_{x, y}^{X \leq z} & & \text { for all } x, y \in I, z \in I^{\geq \max \{x, y\}} \\
& \Leftrightarrow S_{x, y}^{Y \leq z} \leq_{\star} S_{x, y}^{X \leq z} & & \text { for all } z \in I, x, y \in I^{\leq z} \\
& \Leftrightarrow X^{\leq z} \leq_{\star \mathrm{lc}} Y^{\leq z} & & \text { for all } z \in I .
\end{aligned}
$$

Lemma 2.2. Let $X=\left(X_{t}\right)_{t \in \Gamma}$ and $Y=\left(Y_{t}\right)_{t \in \Gamma}$ be stochastic processes with state space $I$, $I \subseteq \mathbb{R}$, unbounded from below (i.e. $I \cap(-\infty, z] \neq \varnothing$, for all $z \in \mathbb{R})$, whose paths are a.s. bounded from below on any bounded time interval. Then

$$
X^{\geq z} \leq_{\text {stlc }} Y^{\geq z} \text { for all } z \in I \quad \Longrightarrow X \leq_{\text {stlc }} Y .
$$

Proof. Let $X, Y$, and $I$ be as stated and choose $x, y \in I$ arbitrarily. To simplify the notation, set $X_{0}=Y_{0}=x$ and $X_{0}^{\geq z}=Y_{0}^{\geq z}=x$ for all $z \in I^{\leq x}$. Note that, for $W=X, Y, t \geq 0$, and $z \in I^{\leq x}$,

$$
\begin{aligned}
\mathrm{P}\left(S_{y}^{W}>t\right)-\mathrm{P}\left(R_{z}^{W} \leq t, S_{y}^{W}>t\right) & =\mathrm{P}\left(R_{z}^{W}>t, S_{y}^{W}>t\right) \\
& =\mathrm{P}\left(R_{z}^{W^{\geq z}}>t, S_{y}^{W^{\geq z}}>t\right) \\
& =\mathrm{P}\left(S_{y}^{W^{\geq z}}>t\right)-\mathrm{P}\left(R_{z}^{W^{\geq z}} \leq t, S_{y}^{W^{\geq z}}>t\right) .
\end{aligned}
$$


Moreover, since the paths of $W$ are a.s. bounded from below on any bounded time interval,

$$
\begin{gathered}
\lim _{z \rightarrow-\infty} \mathrm{P}\left(R_{z}^{W} \leq t, S_{y}^{W}>t\right)=\lim _{z \rightarrow-\infty} \mathrm{P}\left(R_{z}^{W} \leq t\right)=0, \\
\lim _{z \rightarrow-\infty} \mathrm{P}\left(R_{z}^{W \geq z} \leq t, S_{y}^{W \geq z}>t\right)=\lim _{z \rightarrow-\infty} \mathrm{P}\left(R_{z}^{W \geq z} \leq t\right)=0 .
\end{gathered}
$$

Thus, by taking the limit as $z \rightarrow-\infty$ in the previous equation, we conclude that

$$
\mathrm{P}\left(S_{y}^{W}>t\right)=\lim _{z \rightarrow-\infty} \mathrm{P}\left(S_{y}^{W \geq z}>t\right), \quad t \geq 0 .
$$

Now suppose that $X^{\geq z} \leq$ stlc $Y^{\geq z}$ for all $z \in I$, meaning that $\mathrm{P}\left(S_{y}^{X^{\geq z}}>t\right) \leq \mathrm{P}\left(S_{y}^{Y \geq z}>t\right)$ for all $z \in I^{\leq x}$ and $t \geq 0$. Using the last equality, it then follows that

$$
\mathrm{P}\left(S_{y}^{X}>t\right)=\lim _{z \rightarrow-\infty} \mathrm{P}\left(S_{y}^{X \geq z}>t\right) \leq \lim _{z \rightarrow-\infty} \mathrm{P}\left(S_{y}^{Y \geq z}>t\right)=\mathrm{P}\left(S_{y}^{Y}>t\right)
$$

for all $x, y \in I$ and $t \geq 0$, i.e. $X \leq_{\text {stlc }} Y$.

A result similar to the restatement of the previous lemma applied to DTMCs was proved in the final part of the proof of Theorem 4.1 of [5], but there the definition of the process formed from $W$ by restricting the state space to $I^{\geq z}$ is slightly different from ours, in that state $z$ was not made absorbing.

In order to integrate the results of Lemmas 2.1 and 2.2 into the next theorem, it is useful to note that if $W=\left(W_{t}\right)_{t \in \Gamma}$ is a stochastic process with ordered state space $I$ and $x, y \in I$ are such that $x<y$, then $\left(W^{\geq x}\right)^{\leq y}=\left(W^{\leq y}\right)^{\geq x}=W^{[x, y]}$, with $W^{[x, y]}$ denoting the process $W$ restricted to the state space $I \cap[x, y]$ in such a way that the states $x$ and $y$ are made absorbing, all states of $W$ smaller than or equal to $x$ are collapsed into state $x$, and all states of $W$ greater than or equal to $y$ are collapsed into state $y$.

Theorem 2.1. Let $X=\left(X_{t}\right)_{t \in \Gamma}$ and $Y=\left(Y_{t}\right)_{t \in \Gamma}$ be stochastic processes with ordered state space $I$, order-isomorphic to a subset of $\mathbb{R}$ unbounded from below, whose paths are a.s. bounded from below on any bounded time interval. Then

$$
X^{[x, y]} \leq_{\text {stlc }} Y^{[x, y]} \text { for all } x, y \in I \text { such that } x \leq y \quad \Longrightarrow \quad X \leq \text { stlc } Y .
$$

The next definition will be useful when we come to state some of the later results of the paper.

Definition 2.2. Let $\triangle$ denote some property, and let $W$ be a stochastic process on an ordered state space $I$. Then $W$ has the lower- $\triangle$ property if and only if the process $W^{\leq x}$ has the $\triangle$ property for all $x \in I$.

We next introduce some notation and properties of the IPICC stochastic orders that are used in the rest of the paper. We let ' $\geq_{\star}$ ' denote the reverse of ' $\leq_{\star}$ ', and let ' $=_{\star}$ ' denote stochastic equality in the $\star$-sense. Order relation symbols are applied to compare either random variables or their associated distribution functions, e.g. $W \leq_{\star} Z$ is equivalent to $F_{W} \leq_{\star} F_{Z}$, for random variables $W$ and $Z$ with respective distribution functions $F_{W}$ and $F_{Z}$.

We recall that, given two random variables $W$ and $Z$, the variable $W$ is said to be smaller than $Z$ in the usual sense, $W \leq_{\text {st }} Z$, if $\mathrm{P}(W \geq x) \leq \mathrm{P}(Z \geq x)$ for all $x \in \mathbb{R}$. Accordingly, given probability vectors $\boldsymbol{p}=\left(p_{i}\right)_{i \in I}$ and $\boldsymbol{q}=\left(q_{i}\right)_{i \in I}$ with indices in an ordered set $I$, we say that $\boldsymbol{p}$ is smaller than $\boldsymbol{q}$ in the usual sense, $\boldsymbol{p} \leq_{\mathrm{st}} \boldsymbol{q}$, if $\sum_{i \geq j} p_{i} \leq \sum_{i \geq j} q_{i}$ for all $j \in I$. 
A stochastic order ' $\leq_{\star}$ ' is closed for convolution if $W_{1}+W_{2} \leq_{\star} Z_{1}+Z_{2}$ for pairs, $\left(W_{1}, W_{2}\right)$ and $\left(Z_{1}, Z_{2}\right)$, of independent random variables such that $W_{i} \leq_{\star} Z_{i}, i=1,2$. As the usual order is closed for convolution and $G_{\text {st }}$ is the set of all increasing functions, it follows that, for nonnegative random variables $W$ and $Z$,

$$
W \leq_{\text {a.s. }} Z \Rightarrow W \leq_{\text {st }} Z \Rightarrow W \leq_{\star} Z \text { for any IPICC order } \star,
$$

where ' $\leq$ a.s.' stands for almost-sure ordering.

Finally, we state an important universal property of integral stochastic orders that is related to their property (also universal) of being closed under mixture - the property MI in [6, Definition 2.4.1].

Lemma 2.3. Let $X$ and $Y$ be two random variables defined on a common product probability space $\Lambda=\Lambda_{1} \times \Lambda_{2}$, with $\Lambda_{i}=\left(\Omega_{i}, \mathcal{F}_{i}, \mathrm{P}_{i}\right), i=1,2$, such that $X\left(\omega_{1}, \cdot\right)$ and $Y\left(\omega_{1}, \cdot\right)$ are random variables on $\Lambda_{2}$ for each $\omega_{1} \in \Omega_{1}$.

Then $X \leq_{\star} Y$ for each integral stochastic order ' $\leq_{\star}$ ' such that

$$
X\left(\omega_{1}, \cdot\right) \leq_{\star} Y\left(\omega_{1}, \cdot\right) \quad \Lambda_{1} \text {-a.s. }
$$

Proof. Let $\leq_{\star}$ be an integral stochastic order with an associated set of functions $G_{\star}$. Then, for $W=X, Y$ and $g \in G_{\star}$, we have

$$
\mathrm{E}_{\Lambda}[g(W)]=\int_{\Omega_{1}} \mathrm{E}_{\Lambda_{2}}\left[g\left(W\left(\omega_{1}, \cdot\right)\right)\right] \mathrm{P}_{1}\left(\mathrm{~d} \omega_{1}\right),
$$

where, for each $\omega_{1} \in \Omega_{1}$,

$$
\mathrm{E}_{\Lambda_{2}}\left[g\left(W\left(\omega_{1}, \cdot\right)\right)\right]=\int_{\Omega_{2}} g\left(W\left(\omega_{1}, \omega_{2}\right)\right) \mathrm{P}_{2}\left(\mathrm{~d} \omega_{2}\right) .
$$

As, in view of (2.2), $\mathrm{E}_{\Lambda_{2}}\left[g\left(X\left(\omega_{1}, \cdot\right)\right)\right] \leq \mathrm{E}_{\Lambda_{2}}\left[g\left(Y\left(\omega_{1}, \cdot\right)\right)\right] \Lambda_{1}$-a.s., it thus follows that $\mathrm{E}_{\Lambda}[g(X)] \leq \mathrm{E}_{\Lambda}[g(Y)]$ for any $g \in G_{\star}$; that is, $X \leq_{\star} Y$, as required.

We end the section with some notation used in the rest of the paper. Given a matrix $\boldsymbol{A}=$ $\left(A_{i k}\right)_{i, k \in I}$, we let $\boldsymbol{A}_{i}$. $=\left(A_{i j}\right)_{j \in I}$ denote the row vector comprising row $i$ of $\boldsymbol{A}$. In addition, if $\boldsymbol{p}$ denotes a probability vector and $Z$ denotes a random variable or distribution, such as the exponential distribution with rate $\alpha(\operatorname{Exp}(\alpha))$, then we let $\boldsymbol{p}^{-1}$ and $Z^{-1}$ respectively denote the generalized inverses of the distribution functions associated with $\boldsymbol{p}$ and $Z$. Moreover, we let $\mathbf{1}_{A}$ denote the indicator function of the statement $A$, i.e. $\mathbf{1}_{A}=1$ if $A$ is true and $\mathbf{1}_{A}=0$ otherwise. Furthermore, we let $I$ be a set that is order-isomorphic to some bounded or unbounded interval of $\mathbb{Z}$, and let $X=\left(X_{t}\right)_{t \in \Gamma}$ and $Y=\left(Y_{t}\right)_{t \in \Gamma}$ be stochastic processes with common state space $I$.

\section{Discrete-time Markov chains}

Irle and Gani [5, Theorem 4.1] asserted that the level-crossing ordering of two skip-free-tothe-right DTMCs in the usual sense follows from the ordering in distribution of their transition probabilities for any common initial state. In this section, we provide a sample-path proof of this result in a slightly more general form, as we only require the slower DTMC involved in the comparison to be skip-free to the right. For convenience, as the result will subsequently be used to prove results involving SMPs, we will denote the DTMCs involved in the result by $\bar{X}$ and $\bar{Y}$. 
Theorem 3.1. Let $\bar{X}$ and $\bar{Y}$ be two DTMCs with state space I and transition probability matrices $\boldsymbol{P}^{X}$ and $\boldsymbol{P}^{Y}$, respectively. If $\bar{X}$ is skip-free to the right and $\boldsymbol{P}_{i}^{X} \leq_{\mathrm{st}} \boldsymbol{P}_{i}^{Y}$. for all $i \in \bar{I}$, then $\bar{X} \leq_{\text {stlc }} \bar{Y}$.

Proof. Let $\bar{X}$ and $\bar{Y}$ be two DTMCs on $I$ (an ordered set order-isomorphic to some bounded or unbounded interval of $\mathbb{Z}$ ) such that $\boldsymbol{P}_{i}^{X} \leq_{\text {st }} \boldsymbol{P}_{i}^{Y}$. for all $i \in \bar{I}$. For $j \in I$, let $F_{j}^{X}$ and $F_{j}^{Y}$ respectively denote the distribution functions associated with $\boldsymbol{P}_{j}^{X}$. and $\boldsymbol{P}_{j}^{Y}$. Note that $F_{j}^{X}(x) \geq F_{j}^{Y}(x)$ for each $j \in \bar{I}$ and $x \in \mathbb{R}$, since $\boldsymbol{P}_{j}^{X} \leq_{\text {st }} \boldsymbol{P}_{j}^{Y}$. for $j \in \bar{I}$. Thus,

$$
\left[F_{j}^{X}\right]^{-1}(p) \leq\left[F_{j}^{Y}\right]^{-1}(p) \text { for all } j \in \bar{I} \text { and } p \in(0,1) .
$$

We must prove that $S_{i, l}^{\bar{Y}} \leq_{\mathrm{st}} S_{i, l}^{\bar{X}}$ for all $i, l \in I$. As the result is necessarily true when $i=\sup I$ and $\sup I \in I$ (since then $S_{\sup I, l}^{\bar{Y}}=S_{\sup I, l}^{\bar{X}}=0$ ), we will consider only the cases in which $i \in \bar{I}$. Thus, we let $i \in \bar{I}$ and $l \in I$ be two arbitrary states and prove that $S_{i, l}^{\bar{Y}} \leq_{\mathrm{st}} S_{i, l}^{\bar{X}}$ by constructing two processes, $\hat{X}$ and $\hat{Y}$, on a common probability space $\Lambda_{1}=\left(\Omega_{1}, \mathcal{F}_{1}, \mathrm{P}_{1}\right)$, such that $\hat{X}=\left.{ }_{\text {st }} \bar{X}\right|_{\bar{X}_{0}=i}, \hat{Y}=\left.{ }_{\text {st }} \bar{Y}\right|_{\bar{Y}_{0}=i}$, and $S_{l}^{\hat{Y}}\left(\omega_{1}\right) \leq S_{l}^{\hat{X}}\left(\omega_{1}\right)$ for all $\omega_{1} \in \Omega_{1}$. The processes $\hat{X}$ and $\hat{Y}$ are constructed from $\left(U_{n}\right)_{n \in \mathbb{N}_{+}}$and $\left(V_{n}\right)_{n \in \mathbb{N}_{+}}$, two independent sequences of independent uniform $(0,1)$ random variables defined on $\Lambda_{1}$.

The construction of $\hat{Y}$ is similar to the usual generation (i.e. simulation) of a DTMC from a sequence of independent and identically distributed uniform random variables, as presented, for example, in [7]. That is, for each $\omega_{1} \in \Omega_{1}$, the sequence $\left(U_{n}\left(\omega_{1}\right)\right)_{n \in \mathbb{N}_{+}}$is used to construct $\hat{Y}\left(\omega_{1}\right)$ recursively in the following way:

$$
\hat{Y}_{0}\left(\omega_{1}\right)=i, \quad \hat{Y}_{m+1}\left(\omega_{1}\right)=\left[F_{\hat{Y}_{m}\left(\omega_{1}\right)}^{Y}\right]^{-1}\left(U_{m+1}\left(\omega_{1}\right)\right), \quad m \in \mathbb{N} .
$$

As regards the construction of $\hat{X}\left(\omega_{1}\right)$, we will use both $\left(U_{n}\left(\omega_{1}\right)\right)_{n \in \mathbb{N}_{+}}$and $\left(V_{n}\left(\omega_{1}\right)\right)_{n \in \mathbb{N}_{+}}$ to simultaneously generate $\hat{X}\left(\omega_{1}\right)$ and an increasing sequence of times $\left(T_{n}\left(\omega_{1}\right)\right)_{n \in \mathbb{N}}$ such that $T_{0}\left(\omega_{1}\right)=0$ and, if $T_{m}\left(\omega_{1}\right)$ is finite for some $m \in \mathbb{N}$,

$$
\begin{aligned}
\hat{X}_{T_{m}}\left(\omega_{1}\right) & =\hat{Y}_{m}\left(\omega_{1}\right), \\
T_{m+1}\left(\omega_{1}\right) & =\inf \left\{n>T_{m}\left(\omega_{1}\right): \hat{X}_{n}\left(\omega_{1}\right) \geq \hat{Y}_{m+1}\left(\omega_{1}\right)\right\},
\end{aligned}
$$

meaning that $\hat{X}_{n}\left(\omega_{1}\right)<\hat{Y}_{m+1}\left(\omega_{1}\right)$ when $T_{m}\left(\omega_{1}\right)<n<T_{m+1}\left(\omega_{1}\right)$. Specifically, we let $\hat{X}_{0}\left(\omega_{1}\right)=i$ and $T_{0}\left(\omega_{1}\right)=0$ and then proceed recursively as follows. When $T_{m}\left(\omega_{1}\right), m \in \mathbb{N}$, is finite, we first let

$$
\hat{X}_{T_{m}+1}\left(\omega_{1}\right)=\left[F_{\hat{X}_{T_{m}}\left(\omega_{1}\right)}^{X}\right]^{-1}\left(U_{m+1}\left(\omega_{1}\right)\right)
$$

and then, starting with $n=T_{m}\left(\omega_{1}\right)+1$ and while $\hat{X}_{n}\left(\omega_{1}\right)<\hat{Y}_{m+1}\left(\omega_{1}\right)$, let

$$
\hat{X}_{n+1}\left(\omega_{1}\right)=\left[F_{\hat{X}_{n}\left(\omega_{1}\right)}^{X}\right]^{-1}\left(V_{n+1}\left(\omega_{1}\right)\right)
$$

and increment $n$ by one unit. If this cycle ends then $T_{m+1}\left(\omega_{1}\right)$ is (set equal to) the value of $n$ at the end of the cycle, $m$ is incremented by one unit, and the procedure repeated. Otherwise, $T_{j}\left(\omega_{1}\right)$ is set equal to $\infty$ for $j \geq m+1$.

Note that, by construction, $\hat{X}=\left.{ }_{\text {st }} \bar{X}\right|_{\bar{X}_{0}=i}$ and $\hat{Y}=\left.{ }_{\text {st }} \bar{Y}\right|_{\bar{Y}_{0}=i}$ and, moreover, since $\bar{X}$ is skipfree to the right, $\hat{X}\left(\omega_{1}\right)$ is skip-free to the right for all $\omega_{1} \in \Omega_{1}$. Furthermore, $T_{m}\left(\omega_{1}\right) \geq m$ for all $m \in \mathbb{N}$ and, if $T_{m}\left(\omega_{1}\right)$ is finite and $m<\inf \left\{n: \hat{Y}_{n}\left(\omega_{1}\right)=\sup I\right\}$, then

$$
\begin{aligned}
\hat{X}_{T_{j}}\left(\omega_{1}\right) & =\hat{Y}_{j}\left(\omega_{1}\right), & & j=0,1, \ldots, m, \\
\hat{X}_{k}\left(\omega_{1}\right) & <\hat{Y}_{j+1}\left(\omega_{1}\right), & & T_{j}\left(\omega_{1}\right)<k<T_{j+1}\left(\omega_{1}\right), j \in\{0,1, \ldots, m\} .
\end{aligned}
$$


We now argue that $S_{l}^{\hat{X}}\left(\omega_{1}\right) \geq S_{l}^{\hat{Y}}\left(\omega_{1}\right)$. As the result is necessarily true when either $i \geq l$ (since then $\left.S_{l}^{\hat{X}}\left(\omega_{1}\right)=S_{l}^{\hat{Y}}\left(\omega_{1}\right)=0\right)$ or $S_{l}^{\hat{X}}\left(\omega_{1}\right)=\infty$, we assume for the rest of the proof that $S_{l}^{\hat{X}}\left(\omega_{1}\right)=n$ for some $n \in \mathbb{N}_{+}$, and let $m<n$ be the natural number such that $T_{m}\left(\omega_{1}\right)<$ $n \leq T_{m+1}\left(\omega_{1}\right)$. Then, in view of (3.1) and the fact that $\hat{X}$ is skip-free to the right, it follows that

$$
S_{l}^{\hat{X}}\left(\omega_{1}\right)=n \geq T_{m}\left(\omega_{1}\right)+1 \geq m+1=S_{l}^{\hat{Y}}\left(\omega_{1}\right) .
$$

Thus, $S_{l}^{\hat{X}}\left(\omega_{1}\right) \geq S_{l}^{\hat{Y}}\left(\omega_{1}\right)$, as required.

The previous result will be used in the next section to provide sample-path-based proofs of results for the level-crossing ordering of SMPs in the IPICC order senses.

\section{Semi-Markov processes}

In this section, we use a sample-path approach to establish sufficient conditions for the level-crossing ordering of two SMPs in the case in which the slower of the compared SMPs is skip-free to the right. The derived results are an extension of [3, Theorem 2.1], which is itself a generalization of [5, Theorem 4.1] (which corresponds to Theorem 3.1 with the stronger assumption that both $\bar{X}$ and $\bar{Y}$ are lower-regular skip-free-to-the-right DTMCs). Irle [3, Theorem 2.1] established that the level-crossing ordering (in the usual sense) of two skip-freeto-the-right SMPs follows from both the ordering in distribution of their transition probabilities from states and the reversed order (in the usual sense) of the holding times in states before the processes make transitions.

It is convenient to introduce some notation for SMPs. Let $\boldsymbol{P}=\left(P_{i j}\right)_{i, j \in I}$ denote a transition probability matrix and $\boldsymbol{F}=\left(F_{(i, j)}\right)_{i, j \in I}$ a matrix of distribution functions such that if $P_{i j}=0$, then $F_{(i, j)}(x)=1, x \in \mathbb{R}_{+}$. We then say that the process $W=\left(W_{t}\right)_{t \in \mathbb{R}_{+}}$with state space $I$ is an SMP with parametrization $(\boldsymbol{P}, \boldsymbol{F})$ if $W_{t}=Z_{n}, S_{n} \leq t<S_{n+1}$, for some Markov renewal process $(Z, S)=\left(Z_{n}, S_{n}\right)_{n \in \mathbb{N}}$ with phase space $I$ and parametrization $(\boldsymbol{P}, \boldsymbol{F})$, i.e.

$$
\mathrm{P}\left(Z_{n+1}=j, S_{n+1}-S_{n} \leq t \mid Z_{n}=i\right)=P_{i j} F_{(i, j)}(t), \quad i, j \in I, t \in \mathbb{R}_{+} .
$$

Thus, $W$ is an SMP with kernel $\boldsymbol{Q}=\boldsymbol{P} \bullet \boldsymbol{F}$, where ' $\bullet$ ' denotes the Schur or element-wise multiplication of matrices.

We next state and prove the main result of the paper, which improves on [3, Theorem 2.1] by removing the stochastic ordering conditions involving the transition probabilities from the highest state (if it exists), removing the lower regularity and the skip-free to the right properties of the faster of the two processes, and relaxing the conditions on the comparison of the times between transitions in $X$ and $Y$ (namely that $F_{(a, b)}^{X} \geq_{\mathrm{st}} F_{(a, c)}^{Y}$ for all $a, b, c \in I$ ) to $F_{(a, b)}^{X} \geq_{\text {st }} F_{(a, c)}^{Y}$ for all $a \in \bar{I}$ and $b, c \in I$ such that $b \leq c$. In fact, we establish an analogous result for the level-crossing ordering of SMPs in the IPICC order senses.

Theorem 4.1. Let $X$ and $Y$ be SMPs with state space I and parametrizations $\left(\boldsymbol{P}^{X}, \boldsymbol{F}^{X}\right)$ and $\left(\boldsymbol{P}^{Y}, \boldsymbol{F}^{Y}\right)$ such that

$$
\boldsymbol{P}_{i .}^{X} \leq_{\mathrm{st}} \boldsymbol{P}_{i .}^{Y}, \quad i \in \bar{I},
$$

and, for some IPICC order $\star$,

$$
F_{(a, b)}^{X} \geq_{\star} F_{(a, c)}^{Y}
$$

holds simultaneously for all $a \in \bar{I}$ and $b, c \in I, b \leq c$, such that $P_{a b}^{X} P_{a c}^{Y}>0$.

If $X$ is skip-free to the right and lower regular, then $X \leq_{\star \mathrm{lc}} Y$. 
Proof. Let $X$ and $Y$ be as stated, with the condition (4.2) holding for some IPICC order $\star$. We must prove that $S_{i, l}^{Y} \leq_{\star} S_{i, l}^{X}$ for all $i, l \in I$. Thus, we let $i, l \in I$ be two arbitrary states and consider first the case in which $X$ is regular; the case in which $X$ is only lower regular will be addressed at the end of the proof. To prove the desired result, we will construct two processes, $X^{\star}$ and $Y^{\star}$, on a common product probability space $(\Omega, \mathcal{F}, \mathrm{P})=\Lambda_{1} \times \Lambda_{2}$, with $\Lambda_{i}=\left(\Omega_{i}, \mathcal{F}_{i}, \mathrm{P}_{i}\right), i=1,2$, such that $X^{\star}=\left.{ }_{\mathrm{st}} X\right|_{X_{0}=i}, Y^{\star}=\left.{ }_{\mathrm{st}} Y\right|_{Y_{0}=i}$, and $S_{l}^{Y^{\star}} \leq_{\star} S_{l}^{X^{\star}}$. For this purpose, we again let $\left(U_{n}\right)_{n \in \mathbb{N}_{+}}$and $\left(V_{n}\right)_{n \in \mathbb{N}_{+}}$denote independent sequences of independent uniform $(0,1)$ random variables defined on $\Lambda_{1}$. Furthermore, we let $\left(A_{n}\right)_{n \in \mathbb{N}_{+}}$and $\left(B_{n}\right)_{n \in \mathbb{N}_{+}}$ denote independent sequences of independent uniform $(0,1)$ random variables defined on $\Lambda_{2}$.

For $\omega=\left(\omega_{1}, \omega_{2}\right) \in \Omega$, we use $\left(U_{n}\left(\omega_{1}\right)\right)_{n \in \mathbb{N}_{+}}$and $\left(V_{n}\left(\omega_{1}\right)\right)_{n \in \mathbb{N}_{+}}$to construct processes $\hat{X}\left(\omega_{1}\right)$ and $\hat{Y}\left(\omega_{1}\right)$ on $\Lambda_{1}$, such that $\hat{X}$ and $\hat{Y}$ are distributed equally to $\left.\bar{X}\right|_{\bar{X}_{0}=i}$ and $\left.\bar{Y}\right|_{\bar{Y}_{0}=i}$ (exactly as in the proof of Theorem 3.1), where $\bar{X}=\left(\bar{X}_{n}\right)_{n \in \mathbb{N}}$ and $\bar{Y}=\left(\bar{Y}_{n}\right)_{n \in \mathbb{N}}$ are embedded DTMCs of Markov renewal processes with parametrizations $\left(\boldsymbol{P}^{X}, \boldsymbol{F}^{X}\right)$ and $\left(\boldsymbol{P}^{Y}, \boldsymbol{F}^{Y}\right)$, respectively. Thus, we will again employ the notation related to $\hat{X}\left(\omega_{1}\right)$ and $\hat{Y}\left(\omega_{1}\right)$ in the proof of Theorem 3.1. At the same time, the sequences $\left(A_{n}\left(\omega_{2}\right)\right)_{n \in \mathbb{N}_{+}},\left(B_{n}\left(\omega_{2}\right)\right)_{n \in \mathbb{N}_{+}}, \hat{X}\left(\omega_{1}\right)=\left(\hat{X}_{n}\left(\omega_{1}\right)\right)_{n \in \mathbb{N}}$, and $\hat{Y}\left(\omega_{1}\right)=\left(\hat{Y}_{n}\left(\omega_{1}\right)\right)_{n \in \mathbb{N}}$ are used to generate the sequences $H^{X^{\star}}(\omega)=\left(H_{n}^{X^{\star}}(\omega)\right)_{n \in \mathbb{N}_{+}}$and $H^{Y^{\star}}(\omega)=\left(H_{n}^{Y^{\star}}(\omega)\right)_{n \in \mathbb{N}_{+}}$, which consist of the time intervals between state transitions in $X^{\star}(\omega)$ and $Y^{\star}(\omega)$, in such a way that $H_{n}^{X^{\star}}$ and $H_{n}^{Y^{\star}}$ have the respective distributions of the corresponding time intervals between state transitions in $X$ and $Y$.

Specifically, we construct $H^{Y^{\star}}(\omega)$ recursively, using the sequences $\left(A_{n}\left(\omega_{2}\right)\right)_{n \in \mathbb{N}_{+}}$and $\left(\hat{Y}_{n}\left(\omega_{1}\right)\right)_{n \in \mathbb{N}}$, as follows:

$$
H_{n+1}^{Y^{\star}}(\omega)=\left[F_{\left(\hat{Y}_{n}, \hat{Y}_{n+1}\right)\left(\omega_{1}\right)}^{Y}\right]^{-1}\left(A_{n+1}\left(\omega_{2}\right)\right), \quad n \in \mathbb{N} .
$$

For the construction of $H^{X^{\star}}(\omega)$, we will use the random sequences $\left(A_{n}\left(\omega_{2}\right)\right)_{n \in \mathbb{N}_{+}}$and $\left(B_{n}\left(\omega_{2}\right)\right)_{n \in \mathbb{N}_{+}}$and the increasing sequence of times $\left(T_{n}\left(\omega_{1}\right)\right)_{n \in \mathbb{N}}$ used, as explained in the proof of Theorem 3.1, to construct $\hat{X}\left(\omega_{1}\right)$. Starting with $m=0$ and $T_{0}\left(\omega_{1}\right)=0$, we proceed recursively, as follows. When $T_{m}\left(\omega_{1}\right), m \in \mathbb{N}$, is finite, we let

$$
H_{T_{m}+1}^{X^{\star}}(\omega)=\left[F_{\left(\hat{X}_{T_{m}}, \hat{X}_{T_{m}+1}\right)\left(\omega_{1}\right)}^{X}\right]^{-1}\left(A_{m+1}\left(\omega_{2}\right)\right)
$$

and, for those $n$ such that $T_{m}\left(\omega_{1}\right)<n<T_{m+1}\left(\omega_{1}\right)$, we let

$$
H_{n+1}^{X^{\star}}(\omega)=\left[F_{\left(\hat{X}_{n}, \hat{X}_{n+1}\right)\left(\omega_{1}\right)}^{X}\right]^{-1}\left(B_{n+1}\left(\omega_{2}\right)\right)
$$

and increment $m$ by one unit. Finally, we define $X^{\star}(\omega)$ and $Y^{\star}(\omega)$ by letting

$$
W_{t}^{\star}(\omega)=\hat{W}_{n}\left(\omega_{1}\right), \quad S_{n}^{W^{\star}}(\omega) \leq t<S_{n+1}^{W^{\star}}(\omega), W=X, Y
$$

with $S_{0}^{W^{\star}}(\omega)=0$ and $S_{m+1}^{W^{\star}}(\omega)=S_{m}^{W^{\star}}(\omega)+H_{m+1}^{W^{\star}}(\omega), m \in \mathbb{N}$.

Note that we have $X^{\star}=\left.{ }_{\mathrm{st}} X\right|_{X_{0}=i}$ and $Y^{\star}=\left._{\mathrm{st}} Y\right|_{Y_{0}=i}$, by construction, and (3.1) holds, meaning that

$$
\hat{X}_{T_{n}}\left(\omega_{1}\right)=\hat{Y}_{n}\left(\omega_{1}\right) \wedge \hat{X}_{T_{n}+1}\left(\omega_{1}\right) \leq \hat{Y}_{n+1}\left(\omega_{1}\right) \text { for } \omega_{1} \in \Omega_{1} \text { and } n<N^{\hat{Y}}\left(\omega_{1}\right),
$$

where $N^{\hat{Y}}\left(\omega_{1}\right)=\inf \left\{m: \hat{Y}_{m}\left(\omega_{1}\right)=\sup I\right\}$. Since $\left(A_{n+1}\right)_{n \in \mathbb{N}}$ is a sequence of independent and identically uniform $(0,1)$-distributed random variables defined on $\Lambda_{2}$, the random variables $H_{T_{n}+1}^{X^{\star}}\left(\omega_{1}, \cdot\right)$ and $H_{n+1}^{Y^{\star}}\left(\omega_{1}, \cdot\right), n<N^{\hat{Y}}\left(\omega_{1}\right)$, are independent and have respective distribution 
functions $F_{\left(\hat{X}_{T_{n}}, \hat{X}_{T_{n}+1}\right)\left(\omega_{1}\right)}^{X}$ and $F_{\left(\hat{Y}_{n}, \hat{Y}_{n+1}\right)\left(\omega_{1}\right)}^{Y}$, in view of (4.4) and (4.3). By (4.7) and (4.2), this
implies that

$$
H_{T_{n}+1}^{X^{\star}}\left(\omega_{1}, \cdot\right) \geq_{\star} H_{n+1}^{Y^{\star}}\left(\omega_{1}, \cdot\right) \quad \text { for } \omega_{1} \in \Omega_{1} \text { and } n<N^{\hat{Y}}\left(\omega_{1}\right),
$$

and, as the IPICC orderings are closed for convolution,

$$
\sum_{n=0}^{k} H_{T_{n}+1}^{X^{\star}}\left(\omega_{1}, \cdot\right) \geq_{\star} \sum_{n=0}^{k} H_{n+1}^{Y^{\star}}\left(\omega_{1}, \cdot\right), \quad \omega_{1} \in \Omega_{1}, k<N^{\hat{Y}}\left(\omega_{1}\right) .
$$

Now assume that $X$ is regular. Let $\Omega_{1}^{(l)}=\left\{\omega_{1} \in \Omega_{1}: S_{l}^{\hat{X}}\left(\omega_{1}\right)<\infty\right\}$ and $\Omega^{(l)}=\Omega_{1}^{(l)} \times \Omega_{2}$. As, by construction, $S_{l}^{X^{\star}}(\omega)<\infty$ for any $\omega$ such that $S_{l}^{\hat{X}}\left(\omega_{1}\right)<\infty$, it follows that $S_{l}^{X^{\star}}(\omega)<\infty$ for any $\omega \in \Omega^{(l)}$. Furthermore, as $X^{\star}$ is regular and $S_{l}^{\hat{X}}\left(\omega_{1}\right)=\infty$ for all $\omega_{1} \in \Omega_{1} \backslash \Omega_{1}^{(l)}$, we conclude that $S_{l}^{X^{\star}}(\omega)=\infty$ for almost any $\omega \in \Omega \backslash \Omega^{(l)}$. Thus,

$$
\infty=S_{l}^{X^{\star}}(\omega) \geq S_{l}^{Y^{\star}}(\omega) \text { for almost any } \omega \in \Omega \backslash \Omega^{(l)}=\left(\Omega_{1} \backslash \Omega_{1}^{(l)}\right) \times \Omega_{2} .
$$

As $S_{l}^{X^{\star}}(\omega) \geq S_{l}^{Y^{\star}}(\omega)$ is necessarily true when $i \geq l$ (since then $S_{l}^{X^{\star}}(\omega)=S_{l}^{Y^{\star}}(\omega)=0$ ), we now assume that $i<l$ and $\omega_{1} \in \Omega_{1}^{(l)}$. Then, by construction and in view of (3.1), it follows that

$$
1 \leq S_{l}^{\hat{Y}}\left(\omega_{1}\right) \leq T_{S_{l}^{\hat{Y}}-1}\left(\omega_{1}\right)+1 \leq S_{l}^{\hat{X}}\left(\omega_{1}\right)<\infty .
$$

Thus, using (2.1), (4.8), and the fact that $S_{l}^{X^{\star}}(\omega)=\sum_{n=1}^{S_{l}^{\hat{X}}\left(\omega_{1}\right)} H_{n}^{X^{\star}}(\omega)$, we conclude that

$$
\begin{aligned}
S_{l}^{X^{\star}}(\omega) \geq & \sum_{n=1}^{T_{S_{l}^{\hat{Y}}\left(\omega_{1}\right)-1}+1} H_{n}^{X^{\star}}(\omega) \\
= & \sum_{m=0}^{S_{l}^{\hat{Y}}\left(\omega_{1}\right)-2} \sum_{n=T_{m}\left(\omega_{1}\right)+1}^{T_{m+1}\left(\omega_{1}\right)} H_{n}^{X^{\star}}(\omega)+H_{T_{l}^{\hat{Y}}-1}^{X^{\star}+1}(\omega) \\
\geq & \sum_{m=0}^{S_{l}^{\hat{Y}}\left(\omega_{1}\right)-1} H_{T_{m}+1}^{X^{\star}}\left(\omega_{1}, \cdot\right) \\
\geq & \sum_{m=0}^{S_{l}^{\hat{Y}}\left(\omega_{1}\right)-1} H_{m+1}^{Y^{\star}}\left(\omega_{1}, \cdot\right) \\
= & S_{l}^{Y^{\star}}\left(\omega_{1}, \cdot\right) .
\end{aligned}
$$

Accordingly, in view of (2.1) and the results obtained upon assuming that $X$ is regular, we conclude that $S_{l}^{X^{\star}}\left(\omega_{1}, \cdot\right) \geq_{\star} S_{l}^{Y^{\star}}\left(\omega_{1}, \cdot\right) \Lambda_{1}$-a.s. By Lemma 2.3 , this implies that $S_{l}^{X^{\star}} \geq_{\star} S_{l}^{Y^{\star}}$, which is equivalent to having $S_{i, l}^{Y} \leq_{\star} S_{i, l}^{X}$, as required.

If, instead of being regular, the process $X$ is only lower regular, then $X \leq m$ is regular for all $m \in I$. By applying this result to the regular processes $X^{\leq m}$ and $Y \leq m, m \in I$, instead of the processes $X$ and $Y$, we conclude that $X^{\leq m} \leq_{\star l c} Y^{\leq m}$ for all $m \in I$ when (4.1) and (4.2) hold. In view of Lemma 2.1, this is equivalent to having $X \leq_{\star l c} Y$, which concludes the proof of the theorem. 
As we state in the next result, the conditions (4.2) in Theorem 4.1 - on the stochastic ordering of the holding times between transitions - can be further relaxed, at the cost of obtaining conditions that are more difficult to check.

Theorem 4.2. Let $X$ and $Y$ be SMPs with state space $I$ and parametrizations $\left(\boldsymbol{P}^{X}, \boldsymbol{F}^{X}\right)$ and $\left(\boldsymbol{P}^{Y}, \boldsymbol{F}^{Y}\right)$, respectively, such that both (4.1) holds, i.e. $\boldsymbol{P}_{i}^{X} \leq_{\mathrm{st}} \boldsymbol{P}_{i}^{Y}, i \in \bar{I}$, and, for some IPICC order $\star$, the condition

$$
F_{(a, b)}^{X} \oplus F_{(b, b+1)}^{X} \oplus F_{(b+1, b+2)}^{X} \oplus \cdots \oplus F_{(\min (c, a+1)-1, \min (c, a+1))}^{X} \geq_{\star} F_{(a, c)}^{Y},
$$

where ' $\oplus$ ' denotes convolution, holds simultaneously for all $a \in \bar{I}$ and $b, c \in I, b \leq c$, such that

$$
P_{a c}^{Y}>0 \quad \text { and } \quad P_{a b}^{X} \prod_{k=b+1}^{\min (c, a+1)} P_{k-1, k}^{X}>0 .
$$

If $X$ is skip-free to the right and lower regular, then $X \leq_{\star \mathrm{lc}} Y$.

Proof. Let $X$ and $Y$ be as stated, with the condition (4.10) holding for some IPICC order $\star$. We must prove that $S_{i, l}^{Y} \leq_{\star} S_{i, l}^{X}$ for all $i, l \in I$. To this end, we let $i, l \in I$ be two arbitrary states and construct two processes, $X^{\star}$ and $Y^{\star}$, on a common product probability space

$$
\Lambda=(\Omega, \mathcal{F}, P)=\Lambda_{1} \times \Lambda_{2} \times \Lambda_{3}
$$

with $\Lambda_{i}=\left(\Omega_{i}, \mathcal{F}_{i}, \mathrm{P}_{i}\right), i=1,2,3$, such that $X^{\star}=\left.{ }_{\mathrm{st}} X\right|_{X_{0}=i}, Y^{\star}=\left.{ }_{\mathrm{st}} Y\right|_{Y_{0}=i}$, and $S_{l}^{Y^{\star}} \leq_{\star} S_{l}^{X^{\star}}$.

As in Theorem 4.1 , let $\left(U_{n}\right)_{n \in \mathbb{N}_{+}}$and $\left(V_{n}\right)_{n \in \mathbb{N}_{+}}$be independent sequences of independent uniform $(0,1)$ random variables defined on $\Lambda_{1}$, and let $\left(A_{n}\right)_{n \in \mathbb{N}_{+}}$and $\left(B_{n}\right)_{n \in \mathbb{N}_{+}}$be independent sequences of independent uniform $(0,1)$ random variables defined on $\Lambda_{2}$. Moreover, let

$$
\boldsymbol{R}_{(m, t)}^{(a, b, c)}=\left(R_{(m, t, 0)}^{(a, b, c)}, R_{(m, t, 1)}^{(a, b, c)}, \ldots, R_{(m, t, \min (c, a+1)-b)}^{(a, b, c)}\right)
$$

where $m \in \mathbb{N}_{+}, t \in \mathbb{R}_{+}$, and $a, b, c \in I, b \leq c$, be independent random quantities defined on $\Lambda_{3}$ such that

$\boldsymbol{R}_{(m, t)}^{(a, b, c)}={ }_{\mathrm{st}}\left[\left(Z_{(a, b)}^{X}, Z_{(b, b+1)}^{X}, \ldots, Z_{(\min (c, a+1)-1, \min (c, a+1))}^{X}\right) \mid Z_{(a, b)}^{X}+\sum_{j=b+1}^{\min (c, a+1)} Z_{(j-1, j)}^{X}=t\right]$

where the $Z_{(j, k)}^{X}$ are independent random variables with distribution functions $F_{(j, k)}^{X}$.

For $\omega=\left(\omega_{1}, \omega_{2}, \omega_{3}\right) \in \Omega$, we use $\left(U_{n}\left(\omega_{1}\right)\right)_{n \in \mathbb{N}_{+}}$and $\left(V_{n}\left(\omega_{1}\right)\right)_{n \in \mathbb{N}_{+}}$to construct processes $\hat{X}\left(\omega_{1}\right)$ and $\hat{Y}\left(\omega_{1}\right)$ on $\Lambda_{1}$, and use $\left(A_{n}\left(\omega_{2}\right)\right)_{n \in \mathbb{N}_{+}}$along with $\hat{Y}\left(\omega_{1}\right)=\left(\hat{Y}_{n}\left(\omega_{1}\right)\right)_{n \in \mathbb{N}}$ to construct a process $H^{Y^{\star}}(\omega)=\left(H_{n}^{Y^{\star}}(\omega)\right)_{n \in \mathbb{N}_{+}}$, exactly as in the proof of Theorem 4.1. For the construction of $H^{X^{\star}}(\omega)=\left(H_{n}^{X^{\star}}(\omega)\right)_{n \in \mathbb{N}_{+}}$we will use the random sequences $\left(A_{n}\left(\omega_{2}\right)\right)_{n \in \mathbb{N}_{+}}$ and $\left(B_{n}\left(\omega_{2}\right)\right)_{n \in \mathbb{N}_{+}}$, the random variables $\boldsymbol{R}_{(m, t)}^{(a, b, c)}\left(\omega_{3}\right)$, and the increasing sequence of times $\left(T_{n}\left(\omega_{1}\right)\right)_{n \in \mathbb{N}}$ used to construct $\hat{X}\left(\omega_{1}\right)$ (see the proof of Theorem 3.1).

Starting with $m=0$ and $T_{0}\left(\omega_{1}\right)=0$, we proceed recursively, as follows. When $T_{m}\left(\omega_{1}\right)$ is finite, we let

$$
M_{m}\left(\omega_{1}\right)=\min \left(\hat{Y}_{m+1}\left(\omega_{1}\right), \hat{X}_{T_{m}}\left(\omega_{1}\right)+1\right)-\hat{X}_{T_{m}+1}\left(\omega_{1}\right)
$$

and define

$$
I_{m, k}^{\star}\left(\omega_{1}\right)=\inf \left\{n \geq T_{m}\left(\omega_{1}\right)+1: \hat{X}_{n}\left(\omega_{1}\right) \geq \hat{X}_{T_{m}+1}\left(\omega_{1}\right)+k\right\}
$$


for $k=0,1, \ldots, M_{m}\left(\omega_{1}\right)$. Note that $I_{m, k}^{\star}\left(\omega_{1}\right)$ is the first time after $T_{m}\left(\omega_{1}\right)$ at which $\hat{X}\left(\omega_{1}\right)$ reaches the level $\hat{X}_{T_{m}+1}\left(\omega_{1}\right)+k$ and, since $\hat{X}\left(\omega_{1}\right)$ is skip-free to the right, $I_{m, k}^{\star}\left(\omega_{1}\right)$ is increasing in $k$ and takes values in $\left\{T_{m}\left(\omega_{1}\right)+1, T_{m}\left(\omega_{1}\right)+2, \ldots, T_{m+1}\left(\omega_{1}\right)\right\}$. Then, with

$$
(a, b, c)=\left(\hat{X}_{T_{m}}\left(\omega_{1}\right), \hat{X}_{T_{m}+1}\left(\omega_{1}\right), \hat{Y}_{m+1}\left(\omega_{1}\right)\right)
$$

we consecutively let

$$
\begin{gathered}
C_{m+1}\left(\omega_{1}, \omega_{2}\right)=\left[F_{(a, b)}^{X} \oplus F_{(b, b+1)}^{X} \oplus \cdots \oplus F_{(\min (c, a+1)-1, \min (c, a+1))}^{X}\right]^{-1}\left(A_{m+1}\left(\omega_{2}\right)\right), \\
\left(H_{I_{m, 0}^{\star}\left(\omega_{1}\right)}^{X^{\star}}(\omega), H_{I_{m, 1}^{\star}\left(\omega_{1}\right)}^{X^{\star}}(\omega), \ldots, H_{I_{m, M_{m}}^{\star}\left(\omega_{1}\right)}^{X^{\star}}(\omega)\right)=\boldsymbol{R}_{\left(m, C_{m+1}\left(\omega_{1}, \omega_{2}\right)\right)}^{(a, b, c)}\left(\omega_{3}\right),
\end{gathered}
$$

which specifies the construction of $H_{n}^{X^{\star}}(\omega)$ for

$$
n \in I_{m}^{\star}\left(\omega_{1}\right):=\bigcup_{k=0}^{M_{m}\left(\omega_{1}\right)}\left\{I_{m, k}^{\star}\left(\omega_{1}\right)\right\}
$$

For $n$ such that $T_{m}\left(\omega_{1}\right) \leq n<T_{m+1}\left(\omega_{1}\right)$ and $(n+1) \notin I_{m}^{\star}\left(\omega_{1}\right)$, we instead make the assignment (4.5). After doing this, we increment $m$ by one unit and continue with the recursion procedure. Finally, we define $X^{\star}(\omega)$ and $Y^{\star}(\omega)$ as in the proof of Theorem 4.1 (see (4.6)).

Note that, by construction, we have $X^{\star}=\left.{ }_{\mathrm{st}} X\right|_{X_{0}=i}$ and $Y^{\star}=\left._{\mathrm{st}} \quad Y\right|_{Y_{0}=i}$ and, as in Theorem 4.1, (4.7) holds. Since $\left(A_{n+1}\right)_{n \in \mathbb{N}}$ is a sequence of independent and identically uniform $(0,1)$-distributed random variables defined on $\Lambda_{2}$, the random variables $C_{m+1}\left(\omega_{1}, \cdot\right)$, $m<N^{\hat{Y}}\left(\omega_{1}\right)$, are independent and have distribution functions

$$
F_{(a, b)}^{X} \oplus F_{(b, b+1)}^{X} \oplus F_{(b+1, b+2)}^{X} \oplus \cdots \oplus F_{(\min (c, a+1)-1, \min (c, a+1))}^{X},
$$

with

$$
(a, b, c)=\left(\hat{X}_{T_{m}}\left(\omega_{1}\right), \hat{X}_{T_{m}+1}\left(\omega_{1}\right), \hat{Y}_{m+1}\left(\omega_{1}\right)\right),
$$

whereas, by the same line of reasoning, the random variables $H_{m+1}^{Y^{\star}}\left(\omega_{1}, \cdot\right), m<N^{\hat{Y}}\left(\omega_{1}\right)$, are independent and have distribution functions $F_{\left(\hat{Y}_{m}, \hat{Y}_{m+1}\right)\left(\omega_{1}\right)}^{Y}=F_{(a, c)}^{Y}$. By (4.7), (4.11), (4.3), and (4.10), this implies that

$$
C_{m+1}\left(\omega_{1}, \cdot\right) \geq_{\star} H_{m+1}^{Y^{\star}}\left(\omega_{1}, \cdot\right) \quad \text { for } \omega_{1} \in \Omega_{1} \text { and } m<N^{\hat{Y}}\left(\omega_{1}\right),
$$

and that

$$
\sum_{m=0}^{k} C_{m+1}\left(\omega_{1}, \cdot\right) \geq_{\star} \sum_{m=0}^{k} H_{m+1}^{Y^{\star}}\left(\omega_{1}, \cdot\right), \quad \omega_{1} \in \Omega_{1}, k<N^{\hat{Y}}\left(\omega_{1}\right),
$$

since the IPICC orderings are closed for convolution.

We now assume that $X$ is regular. For $l \in I$, let $\Omega_{1}^{(l)}=\left\{\omega_{1} \in \Omega_{1}: S_{l}^{\hat{X}}\left(\omega_{1}\right)<\infty\right\}$ and $\Omega^{(l)}=\Omega_{1}^{(l)} \times \Omega_{2} \times \Omega_{3}$. As, by construction, $S_{l}^{X^{\star}}(\omega)<\infty$ for any $\omega$ such that $S_{l} \hat{X}\left(\omega_{1}\right)<\infty$, it follows that $S_{l}^{X^{\star}}(\omega)<\infty$ for any $\omega \in \Omega^{(l)}$. Moreover, as $X^{\star}$ is regular and $S_{l}^{\hat{X}}\left(\omega_{1}\right)=\infty$ for all $\omega_{1} \in \Omega_{1} \backslash \Omega_{1}^{(l)}$, we conclude that $S_{l}^{X^{\star}}(\omega)=\infty$ for almost any $\omega \in \Omega \backslash \Omega^{(l)}$. Thus, $\infty=S_{l}^{X^{\star}}(\omega) \geq S_{l}^{Y^{\star}}(\omega)$ for almost any $\omega \in \Omega \backslash \hat{\Omega}^{(l)}=\left(\Omega_{1} \backslash \Omega_{1}^{(l)}\right) \times \Omega_{2} \times \Omega_{3}$. As 
$S_{l}^{X^{\star}}(\omega) \geq S_{l}^{Y^{\star}}(\omega)$ is necessarily true when $i \geq l$ (since then $S_{l}^{X^{\star}}(\omega)=S_{l}^{Y^{\star}}(\omega)=0$ ), in the remainder of the proof we assume that $i<l$ and $\omega_{1} \in \Omega_{1}^{(l)}$, in which case $T_{m}\left(\omega_{1}\right) \geq m$ for all $m \in \mathbb{N}, S_{l}^{\hat{X}}\left(\omega_{1}\right) \geq T_{S_{l}^{\hat{Y}}-1}\left(\omega_{1}\right)+1$, and $S_{l}^{\hat{Y}}\left(\omega_{1}\right)>0$.

Since

$$
S_{l}^{X^{\star}}\left(\omega_{1}, \cdot\right)=\sum_{n=1}^{S_{l}^{\hat{X}}\left(\omega_{1}\right)} H_{n}^{X^{\star}}\left(\omega_{1}, \cdot\right),
$$

by using (4.12) we conclude that

$$
\begin{aligned}
S_{l}^{X^{\star}}\left(\omega_{1}, \cdot\right) & =\sum_{m=0}^{S_{l}^{\hat{Y}}\left(\omega_{1}\right)-2} \sum_{n=T_{m}\left(\omega_{1}\right)+1}^{T_{m+1}\left(\omega_{1}\right)} H_{n}^{X^{\star}}\left(\omega_{1}, \cdot\right)+\sum_{n=T_{S_{l}^{\hat{Y}}-1}\left(\omega_{1}\right)+1}^{S_{l}^{\hat{X}}\left(\omega_{1}\right)} H_{n}^{X^{\star}}(\omega) \\
& \geq \sum_{m=0}^{S_{l}^{\hat{Y}}\left(\omega_{1}\right)-1} \sum_{n \in I_{m}^{\star}\left(\omega_{1}\right)} H_{n}^{X^{\star}}\left(\omega_{1}, \cdot\right) \\
& =\sum_{m=0}^{S_{l}^{\hat{Y}}\left(\omega_{1}\right)-1} C_{m+1}\left(\omega_{1}, \cdot\right) \\
& \sum_{\star} \sum_{m=0}^{\hat{Y}\left(\omega_{1}\right)-1} H_{m+1}^{Y^{\star}}\left(\omega_{1}, \cdot\right) \\
& =S_{l}^{Y^{\star}}\left(\omega_{1}, \cdot\right) .
\end{aligned}
$$

By repeating the argument following (4.9) in the proof of Theorem 4.1, we find that Theorem 4.2 now follows.

\section{Continuous-time Markov chains}

In this section, we derive sufficient conditions for the level-crossing ordering (in the usual sense) of CTMCs for the case in which the slower of the compared CTMCs is skip-free to the right. Similar to the sufficient conditions derived in the previous section for the comparison of SMPs, these conditions for CTMCs involve only comparisons of transition rates and probabilities for common departure states.

We recall that a CTMC $W$ with state space $I$ and generator matrix $\boldsymbol{Q}=\left(q_{i j}\right)_{i, j \in I}$, whose corresponding transition rate from state $i$ is $q_{i} \equiv-q_{i i}=\sum_{j \neq i} q_{i j}$, may be interpreted as an SMP with one-step embedded transition probability matrix $\boldsymbol{P}=\left(P_{i j}\right)_{i, j \in I}$, where $P_{i j}=\left(1-\delta_{i j}\right) q_{i j} / q_{i}$ if $q_{i}>0$ and $P_{i j}=\delta_{i j}$ if $q_{i}=0$, and holding times in state $i$ exponentially distributed with rate $q_{i}$, irrespective of the state visited at the next transition. Moreover, $\sum_{j \geq k} P_{i j}=\mathbf{1}_{\{k \leq i\}}$ if $q_{i}=0$ and

$$
\sum_{j \geq k} P_{i j}= \begin{cases}\sum_{j \geq k} \frac{q_{i j}}{q_{i}}, & k>i, \\ 1+\sum_{j \geq k} \frac{q_{i j}}{q_{i}}, & k \leq i,\end{cases}
$$


otherwise. In addition, if $W$ is a CTMC with ordered state space $I$ and generator matrix $\boldsymbol{Q}$, and $x, y \in I$ are such that $x \leq y$, then $W^{[x, y]}$ is a CTMC with state space $I^{[x, y]}$ and generator matrix $Q^{W^{[x, y]}}$ such that $q_{i j}^{W^{[x, y]}}=0$ for $i=x, y$ and

$$
q_{i j}^{W^{[x, y]}}= \begin{cases}q_{i j}, & x<i, j<y, \\ \sum_{k \geq y} q_{i k}, & x<i<j=y, \\ \sum_{k \leq x} q_{i k}, & x=j<i<y .\end{cases}
$$

The next result follows from a convenient application of Theorem 4.1 to CTMCs.

Theorem 5.1. Let $X$ and $Y$ be CTMCs with state space I, generator matrices $\boldsymbol{Q}^{X}$ and $\boldsymbol{Q}^{Y}$, vectors $\boldsymbol{q}^{X}$ and $\boldsymbol{q}^{Y}$ of transition rates from states, and one-step embedded transition probability matrices $\boldsymbol{P}^{X}$ and $\boldsymbol{P}^{Y}$, respectively. Then $X \leq{ }_{\text {stlc }} Y$ if either

(i) $X$ is skip-free to the right and lower regular, and

$$
q_{i}^{X} \leq q_{i}^{Y} \quad \text { and } \quad \boldsymbol{P}_{i .}^{X} \leq_{\mathrm{st}} \boldsymbol{P}_{i}^{Y} . \text { for all } i \in \bar{I}
$$

or

(ii) $X$ and $Y$ are lower regular, $X$ is skip-free to the right, and there exists a vector $\bar{\alpha}=\left(\alpha_{i}\right)_{i \in \bar{I}}$, with entries in $(0,1]$, such that

$$
\sum_{n \geq m} q_{i n}^{X} \leq \alpha_{i} \sum_{n \geq m} q_{i n}^{Y} \quad \text { for all } i \in \bar{I} \text { and } m \in I .
$$

Proof. Let $X$ and $Y$ be CTMCs as stated. We treat the cases (i) and (ii) separately.

Case (i). Suppose that $X$ is skip-free to the right and lower regular, and that $X$ and $Y$ are such that (5.2) holds. Then, as (5.2) is a direct translation of the conditions of Theorem 4.1, for the usual stochastic order, with the CTMCs $X$ and $Y$ viewed as SMPs, it follows that $X \leq$ stlc $Y$.

Case (ii). Suppose that $X$ and $Y$ are lower-regular CTMCs and that (5.3) holds for a given vector $\overline{\boldsymbol{\alpha}}=\left(\alpha_{i}\right)_{i \in \bar{I}}, \alpha_{i} \in(0,1]$. For each $x, y \in I$ such that $x \leq y$, the processes $X^{[x, y]}$ and $Y^{[x, y]}$ are CTMCs with respective generator matrices $\boldsymbol{Q}^{X^{[x, y]}}$ and $\boldsymbol{Q}^{Y^{[x, y]}}$ such that, in view of (5.1), $\sum_{n \geq m} q_{i n}^{X^{[x, y]}} \leq \alpha_{i} \sum_{n \geq m} q_{i n}^{Y^{[x, y]}}$ for all $i \in I^{[x, y)}$ and $m \in I^{[x, y]}$. Thus, by appealing to Theorem 2.1 we may, without loss of generality, assume that $X$ and $Y$ have a finite state space, i.e. $I$ is bounded. As a consequence, $X$ and $Y$ are uniformizable. Thus, we consider the uniformized CTMCs $X$ and $Y$ with state-dependent uniformization rates in such a way that if, at a certain instant, $X$ and $Y$ are in states $j$ and $k$, respectively, then $X$ and $Y$ are uniformized with rates $\alpha_{j} \lambda_{j}$ and $\lambda_{k}$, respectively, with $\lambda_{n} \geq \max \left\{q_{n}^{Y}, q_{n}^{X} / \alpha_{n}\right\}$ being positive.

More specifically, we let $\lambda=\left(\lambda_{n}\right)_{n \in I}$ and consider the uniformized CTMCs $X$ and $Y$ with one-step embedded transition probability matrices

$$
\hat{\boldsymbol{P}}^{X}=\boldsymbol{I}+[\operatorname{diag}(\boldsymbol{\alpha} \bullet \boldsymbol{\lambda})]^{-1} \boldsymbol{Q}^{X} \quad \text { and } \quad \hat{\boldsymbol{P}}^{Y}=\boldsymbol{I}+[\operatorname{diag}(\boldsymbol{\lambda})]^{-1} \boldsymbol{Q}^{Y},
$$

where $\boldsymbol{\alpha}=\left(\alpha_{i}\right)_{i \in I}$ with $\alpha_{\text {sup } I}$ chosen arbitrarily in $(0,1]$ if $I$ is bounded. As the conditions (5.3) imply that $\hat{\boldsymbol{P}}_{i .}^{X} \leq_{\text {st }} \hat{\boldsymbol{P}}_{i}^{Y}$ for all $i \in \bar{I}$, and $\alpha_{i} \lambda_{i} \leq \lambda_{i}$ for all $i \in \bar{I}$, the result of case (i) for the uniformized CTMCs $X$ and $Y$ gives $X \leq_{\text {stlc }} Y$, as required. 
In the proof of the previous result, we have used state-dependent uniformization rates for both CTMCs under consideration. This is similar to an idea suggested in a personal communication by Irle [4]. For uniformizable skip-free-to-the-right CTMCs, Theorem 5.1 generalizes [2, Theorem 3.1] in two ways: it requires only the slower CTMC to be skip-free to the right, rather than both of them, and relaxes the conditions

$$
\sum_{n \geq m} q_{i n}^{X} \leq \alpha \sum_{n \geq m} q_{i n}^{Y} \quad \text { for all } i \in \bar{I} \text { and } m \in I,
$$

for some constant $\alpha \in(0,1]$, to (5.3).

\section{Acknowledgements}

This research was supported in part by Programa Operacional 'Ciência, Tecnologia, Inovação' (POCTI) of the Fundação para a Ciência e a Tecnologia (FCT) and cofinanced by the European Community fund FEDER, the projects POSI/EIA/60061/2004 and POCI/MAT/ 55796/2004, and EuroNGI.

\section{References}

[1] El-Taha, M. and Stidham, S., JR. (1999). Sample-Path Analysis of Queueing Systems. Kluwer, Boston, MA.

[2] Ferreira, F. and Pacheco, A. (2005). Level-crossing ordering of skip-free-to-the-right continuous-time Markov chains. J. Appl. Prob. 42, 52-60.

[3] IrLe, A. (2003). Stochastic ordering for continuous-time processes. J. Appl. Prob. 40, 361-375.

[4] IRLE, A. (2004). Some further thoughts on the level crossing order. Personal communication.

[5] IRLE, A. ANd GAni, J. (2001). The detection of words and an ordering for Markov chains. In Probability, Statistics and Seismology (J. Appl. Prob. Spec. Vol. 38A), Applied Probability Trust, Sheffield, pp. 66-77.

[6] Müller, A. And Stoyan, D. (2002). Comparison Methods for Stochastic Models and Risks. John Wiley, Chichester.

[7] Resnick, S. (1992). Adventures in Stochastic Processes. Birkhäuser, Boston, MA.

[8] Shaked, M. and Shanthikumar, J. G. (1994). Stochastic Orders and Their Applications. Academic Press, San Diego, CA.

[9] Whitт, W. (1986). Stochastic comparisons for non-Markov processes. Math. Operat. Res. 11, 609-618. 